\title{
UNE PERSPECTIVE FAMILIALE
}

\author{
LAURA ENICK \\ Société de schizophrénie de la Saskatchewan
}

\begin{abstract}
Laura Enick a obtenu son diplôme d'infirmière de l'hôpital Grey Nun, à Regina, Saskatchewan en 1958. Elle a travaillé depuis en gériatrie, en neurochirurgie, en ophtalmologie et en gynécologie. Elle est une bénévole active au sein du programme de soins infirmiers à domicile. De plus, Mme Enick démontre des qualités de chef auprès des jeunes. Elle travaille à la Société de schizophrénie de la Saskatchewan, dont elle est présidente provinciale et principale responsable de la défense des droits. Membre du Conseil d'administration de la Société canadienne de schizophrénie pendant cinq ans, elle y a travaillé à la défense des droits, au développement des ressources humaines de mềme qu'au sein de divers comités exécutifs. À titre de membre exécutif de la Société de la schizophrénie de la Saskaichewan, Mme Enick aide les familles des personnes souffrant de cette maladie à s'occuper de leur malade au jour le jour. La Société s'efforce de trowver pour eux les meilleurs régimes de traitement existants quelle que soit la région où ils habitent, et notamment de leur donner accès aux médicaments et aux services de soutien et de prise en charge qui conviennent le mieux à leurs besoins. Ensemble, its luttent contre le manque de coopération et d'engagement, le déni et l'absence de vision. À titre d'organisation, la Société fait des pressions sur le gouvemement provincial pour obtenir les médicaments les plus récents, des lits de soins aigus lorsque cela est nécessaire, des fonds pour la réadaptation et des fonds et des engagements pour des lits de soins de longue durée destinés aux personnes qui ont besoin de soins structurés ou protégés permanents. La Société estime également nécessaire de mettre sur pied un régime de pensions pour invalidité ou un programme d'incitatifs pour le travail à temps partiel à l'intention des personnes souffrans de troubles psychiatriques, afin de les aider à sortir du cycle de la pauvreté. Ces luttes au quotidien visent à améliorer la qualité de vie des personnes souffrant de schizophrénie.
\end{abstract}

La schizophrénie est devenue une réalité dans notre foyer, il y a 11 ans. Au cours de ces années, notre fille a tenté de se suicider cinq fois. Chaque fois, elle a échappé à la mort. Cela lui a coûté ses études secondaires et lui a volé ses années d'adolescence. Elle a aujourd'hui 26 ans. Heureusement pour elle, elle est sortie d'un état prolongé de catatonie il y a cinq ans, et est aujourd'hui mariée et heureuse. Elle occupe un emploi à temps partiel peu stressant au salaire minimum. Nous avons bien sûr dû revoir quelque peu les rêves que nous faisions pour elle lorsqu'elle avait 14 ans, mais sur plusieurs autres plans, elle a dépassé nos attentes.

Parce qu'à titre de mère, je me sens si favorisée, j'ai consacré une bonne partie des six dernières années à conseiller et à aider d'autres familles aux prises avec cette maladie, à faire pression pour que les personnes souffrant de schizophrénie obtiennent de meilleurs services, et à essayer de sensibiliser la population aux maladies mentales les plus graves.

La qualité de vie des parents d'une personne souffrant de maladie mentale grave, comme la schizophrénie, est directement liée à celle de l'être cher qui souffre. S'occuper d'une personne atteinte de maladie physique chronique est sou- 
vent exigeant pour la famille sur les plans physique, affectif et financier. Mais lorsque la maladie en question affecte le cerveau, la famille doit en plus faire face à une situation *sans issuew. Les parents d'une personne atteinte de maladie psychiatrique veulent lui venir en aide et faire pour elle ce qui convient, mais souvent, ils font face à des problèmes d'ordre juridique et éthique. Lorsqu'ils n'arrivent pas à obtenir une aide rapide et opportune pour leur parent malade, ils se retrouvent dans une situation intolérable.

En effet, e'est le même cerveau qui est à l'origine de la maladie qui décide s'il a besoin ou non de se faire soigner. Une étude sur les personnes atteintes de schizophrénie chronique a montré que seulement $13 \%$ d'entre elles étaient vraiment conscientes de souffrir de maladie mentale. Même si l'on sait que la maladie ne perturbe pas l'ensemble du cerveau de ces personnes, on ne dispose pour l'heure d'aucun moyen qui permettrait de déterminer quelles parties du cerveau sont perturbées et lesquelles fonctionnent normalement. Il est absurde d'affirmer que le refus d'une personne de se faire traiter vient de la partie saine de son cerveau. Étonnamment, mềme les personnes qui affirment connaître à fond la maladie et qui sont en mesure de refuser ou d'accepter les demandes de traitement adressées par la famille de la personne atteinte hésitent souvent à donner les soins nécessaires. Cette situation, cette constante incapacité de se faire comprendre et d'obtenir une aide professionnelle efficace, est à elle seule dévastatrice pour les familles.

Bien que la plupart les personnes atteintes de troubles psychiatriques soient non violentes, ce sont leurs parents et leurs proches qui sont les plus susceptibles d'être leurs victimes dès qu'elles adoptent un comportement agressif. Trop souvent, les parents, dont beaucoup sont des personnes âgées, sont tenus en otages dans leur propre maison. La personne malade refuse de se faire traiter et est dans un état explosif. Lorsque la famille obtient l'ordonnance d'un tribunal pour la faire interner, mais que l'hospitalisation ne peut se faire immédiatement ou pour une période assez longue pour que son état se stabilise, elle revient souvent à la maison dans un état encore plus dangereux, blâmant les personnes qui ont demandé l'ordonnance et transformant leur vie en un véritable cauchemar. On ne risque guère d'exagérer en disant qu'il est impossible de vivre une vie familiale normale dans de telles circonstances.

Depuis la création de groupes de soutien à la famille partout au Canada au début des années '80, les familles apprennent graduellement à obtenir l'aide dont elles ont besoin. Au cours des dernières années, avec l'aide financière de Santé Canada, nous avons pu, à titre d'organisation provinciale de la Société de schizophrénie en Saskatchewan, mettre en place des programmes à l'intention des familles des personnes atteintes de schizophrénie, et notamment des parents âgés. Les trois projets suivants en sont de bons exemples.

Le projet What's In a Name: Ce projet consiste en un programme informatique qui imprime le sens du mot schizophrénie et celui du nom du participant sur une petite carte d'affaire. Cela est utile dans les assemblées publiques pour attirer les gens, de façon non menaçante, vers une source d'information sur la schizophrénie. 
Lignes directrices sur la planification successorale et testamentaire à l'intention des parents de personnes souffrant de déficiences psychiatriques: Financé par le Programme d'autonomie des aîné(e)s de Santé Canada, ce projet réunit des parents désireux de planifier l'avenir financier de leurs proches, de mettre en place des ressources communautaires pour résoudre les problèmes, et de favoriser le changement social au besoin. Les documents produits sont utilisés partout au Canada par des avocats et des professionnels de la santé mentale aussi bien que par des familles.

Programme Caring and Sharing: Ce programme qui vient de prendre fin nous a permis de mettre en place des groupes de compréhension et de soutien émotif dans quelque 14 régions importantes de la Saskatchewan, dans le but d'aider les parents de personnes malades à résoudre leurs problèmes, de leur enseigner des habiletés d'adaptation, de leur expliquer, à eux et aux malades, en quoi consiste la maladie, de façon à réduire le stress et la stigmatisation qui y sont associés, et de les aider à partager leur expérience les uns avec les autres. Le but est de continuer à alimenter ces groupes jusqu'à ce qu'ils deviennent autonomes. Cela fait beaucoup pour améliorer la qualité de vie des familles, pour les habiliter à prendre les mesures qui s'imposent, et, en retour, les libérer des sentiments d'impuissance et de désespoir que ne manque pas de provoquer la présence de la schizophrénie chez un membre de la famille.

Toute personne atteinte de maladie mentale doit recevoir un traitement rapide et efficace faisant appel aux meilleurs antipsychotiques, et être encouragée de toutes les façons possibles à se conformer à ce traitement. Si ces personnes pouvaient disposer de logements sürs et autonomes adaptés à leurs besoins, et occuper, lorsque cela est possible, un emploi répondant à leurs capacités et leur apportant la stabilité financière nécessaire pour répondre à leurs besoins et avoir des relations sociales, une grande partie du poids qui pèse sur les épaules des parents serait levée. Ceux-ci pourraient alors vivre leur vie et tenter eux aussi de trouver le bonheur et de combler leurs désirs.

Enfin, le suicide est l'issue la plus dévastatrice de la schizophrénie. En plus de mettre fin à la vie de la personne malade, le suicide a un impact lourd et durable sur la famille. Selon des études récentes, l'incidence du suicide serait de $10 \%$ durant les 10 premières années de la maladie, et de $15 \%$ au cours de la vie. La mort auto-administrée n'est pas la manifestation d'un rejet de la vie, mais plutôt du désir désespéré d'une vie meilleure. À titre de familles de ces personnes, nous nous faisons l'écho de ce désir de tout notre être. À titre de parents, d'époux ou d'épouses, et d'enfants de personnes souffrant de maladies mentales, nous voudrions que ces êtres que nous aimons puissent bénéficier dans la vie des mêmes chances que tout autre membre de la sociêté. 